\title{
Smaller grey matter volumes in the anterior cingulate cortex and greater cerebellar volumes in patients with long-term remission of Cushing's disease: a case-control study
}

\author{
Cornelie D Andela $^{1,2, *}$, Steven J A van der Werff ${ }^{2,3, *}$, J Nienke Pannekoek ${ }^{2,3}$, Susan M van den Berg ${ }^{3}$, \\ Onno C Meijer ${ }^{1,2}$, Mark A van Buchem ${ }^{2,4}$, Serge A R B Rombouts ${ }^{2,4,5}$, Roos C van der Mast ${ }^{3}$, Johannes A Romijn ${ }^{6}$, \\ Jitske Tiemensma $^{7}$, Nienke R Biermasz ${ }^{1,2}$, Nic J A van der Wee ${ }^{2,3}$ and Alberto M Pereira ${ }^{1,2}$ \\ ${ }^{1}$ Department of Endocrinology and Metabolic Diseases, C7-Q, Center for Endocrine Tumors, Leiden University Medical Center, PO Box 9600,2300 RC \\ Leiden, The Netherlands, ${ }^{2}$ Leiden Institute for Brain and Cognition, Leiden, The Netherlands, Departments of ${ }^{3}$ Psychiatry and ${ }^{4}$ Radiology, Leiden University \\ Medical Center, Leiden, The Netherlands, ${ }^{5}$ Institute of Psychology, Leiden University, Leiden, The Netherlands, ${ }^{6}$ Department of Medicine, Academic Medical \\ Center, Amsterdam, The Netherlands and ${ }^{7}$ Psychological Sciences, University of California, Merced, California, USA
}

(Correspondence should be addressed to C D Andela at Department of Endocrinology and Metabolic Diseases, Leiden University Medical Center; Email: c.d.andela@lumc.nl)

*(C D Andela and S J A van der Werff share first authorship)

\begin{abstract}
Objective: Patients with long-term remission of Cushing's disease (CD) have persistent psychological and cognitive impairments. It is unknown whether, and to what extent, these impairments are accompanied by structural abnormalities in the brain. We aim to investigate structural changes in the brain in patients with predominantly long-term remission of $\mathrm{CD}$ and to examine whether these changes are associated with psychological and cognitive dysfunction and clinical severity.

Design: A cross-sectional, case-control study.

Methods: In 25 patients with predominantly long-term remission of CD and 25 matched healthy controls, grey matter volumes in the regions of interest (hippocampus, amygdala, and anterior cingulate cortex (ACC)) and in the whole brain were examined, using 3T magnetic resonance imaging and a voxel-based morphometry approach. Psychological and cognitive functioning were assessed using validated questionnaires and clinical severity was assessed using the Cushing's syndrome severity index.

Results: Compared with controls, patients had smaller grey matter volumes of areas in the ACC (on average $14 \%, P<0.05$ ) and greater volume of the left posterior lobe of the cerebellum (on average $34 \%$, $P<0.05)$. As expected, patients with remitted CD reported more depressive symptoms $(P=0.005)$, more anxiety $(P=0.003)$, more social phobia $(P=0.034)$, more apathy $(P=0.002)$, and more cognitive failure $(P=0.023)$ compared with controls, but the differences in grey matter volumes were not associated with psychological or cognitive measures, nor with clinical severity.

Conclusion: Patients with predominantly long-term remission of CD showed specific structural brain abnormalities, in the presence of psychological dysfunction. Our data form a basis for future work aimed at elucidating the relation of the structural brain abnormalities and the sustained psychological deficits after long-term exposure to high cortisol levels.
\end{abstract}

European Journal of Endocrinology 169 811-819

\section{Introduction}

Cushing's disease (CD) is caused by excessive endogenous cortisol exposure (1). After successful surgical correction of hypercortisolism, the physical, psychological, and cognitive symptoms improve substantially $(2,3)$. However, despite curative treatment of the adenomas per se, multiple physical, psychological, and cognitive complaints may persist and morbidity and mortality remain increased even in the case of long-term remission $(4,5)$.

Cortisol is the main hormonal mediator of the stress response and acts via stimulation of both the mineralocorticoid receptor (MR) and glucocorticoid receptor (GR) in the CNS. Hypothalamic-pituitaryadrenal (HPA)-axis activity is regulated by limbic structures such as the hippocampus and amygdala and the anterior cingulate cortex (ACC) (6). These areas are also important target areas for glucocorticoid hormones via activation of MR and GR. In accordance, long-term exposure to elevated cortisol levels has been linked to functional and structural changes of these limbic structures both in humans and preclinical studies $(7,8)$. For example, prolonged cortisol elevations predict memory dysfunction and reduced volume of the hippocampus and ACC during aging $(9,10)$. Moreover, in patients with Cushing's syndrome, hypercortisolism 
was associated with smaller hippocampal volumes and overall brain atrophy $(11,12,13)$, with increasing hippocampal volumes and improving emotional and cognitive functioning after correction of hypercortisolism $(11,14,15,16,17,18)$.

To date, the long-term effects of chronic overexposure to cortisol, such as in $\mathrm{CD}$, on the brain has been evaluated in only one study (19). In that study, focusing on memory function and hippocampal volume in 33 patients with Cushing's syndrome and 34 matched healthy controls, no overall differences in hippocampal volume between patients and controls were found (19). However, there was a considerable heterogeneity within the patient group in terms of disease status and treatment. Both patients with active $\mathrm{CD}$ and patients with CD in remission, with either pituitary or adrenal disease, were included and analyzed as one group (19), precluding definite conclusions. Furthermore, volumetric analyses were limited to the hippocampus and did not include other brain regions known to be important in emotional and cognitive functioning.

Recently, we performed a large cross-sectional study in a well-characterized cohort of patients with long-term biochemical remission, i.e. successful treatment for CD. We found a decreased quality of life $(20,21)$, a higher prevalence of psychopathology (e.g. depression, anxiety, and apathy) (22), maladaptive personality traits (22), and subtle cognitive impairments (23) despite long-term cure. The results of these studies suggest irreversible effects of longer periods with glucocorticoid excess on brain function and possibly brain structure. These findings were associated with clinical characteristics (e.g. hydrocortisone dependency).

The primary aim of the present cross-sectional study was to investigate whether this cohort of patients with predominantly long-term biochemical remission of pituitary-dependent CD shows structural brain abnormalities, using a voxel-based morphometry approach. In particular, given the results of our previous study, we aimed to evaluate structural changes in important cerebral regions of the limbic system, i.e. the hippocampus, the amygdala, and also in a cerebral key region for both cognitive and emotional functioning: the ACC. Furthermore, we performed an explorative whole brain analysis to detect possible structural changes in areas outside these a priori defined regions of interest (ROI). In addition, we aimed to explore associations between structural changes and measures of psychological and cognitive dysfunction and to take clinical characteristics, such as hydrocortisone dependency, into account.

\section{Subjects and methods}

\section{Subjects}

All patients in long-term remission of CD of pituitary origin, monitored at our institute $(n=49)$ and between
18 and 60 years of age, were invited by letter and those who did not respond were contacted by phone. The response rate was $96 \%$ and 31 patients were screened for eligibility. Exclusion criteria were (history of) drug or alcohol abuse, neurological problems, contraindications for undergoing a magnetic resonance imaging (MRI) scan, and left-handedness. A total of $25 \mathrm{CD}$ patients and 25 matched healthy controls were included in this study. All CD patients had been treated by transsphenoidal surgery, two patients $(8 \%)$ additionally underwent bilateral adrenalectomy, whereas six patients (24\%) had received additional radiotherapy. One patient (4\%) used antidepressants. Healthy controls were pairwise matched for gender, age, and education and recruited by advertisements in grocery stores and via Internet. Inclusion criteria for healthy controls were aged between 18 and 60 years, right handedness, no current or prior drug or alcohol abuse, no present and past history of psychiatric or neurological disorders, no use of psychotropic medication, and no contraindications for MRI scanning.

The diagnosis of $\mathrm{CD}$ had been confirmed in all patients. Adrenocorticotrophic hormone (ACTH)dependent Cushing's syndrome had been diagnosed based on internationally agreed guidelines, with clinical manifestations and positive biochemical tests, including increased urinary excretion rates of free cortisol, decreased overnight suppression by dexamethasone (1 mg), and elevated midnight salivary cortisol values. Cure of $\mathrm{CD}$ had been achieved by transsphenoidal surgery and, if necessary, followed by repeated surgery and/or postoperative radiotherapy. Cure of CD was defined by normal overnight suppression of plasma cortisol levels $(<50 \mathrm{nmol} / \mathrm{l})$ after administration of dexamethasone (1 mg) and normal 24-h urinary excretion rates of cortisol $(<220 \mathrm{nmol} / 24 \mathrm{~h})$. Hydrocortisone independency was defined as a normal cortisol response to corticotrophin-releasing hormone $(\mathrm{CRH})$ or insulin tolerance test $(>500 \mathrm{nmol} / \mathrm{l})$. Patients were followed at our department with yearly intervals, and pituitary hormone substitution was prescribed in accordance with the results of the yearly evaluation. In patients who were glucocorticoid dependent after treatment, recovery of the pituitary-adrenal axis was tested twice a year. The dose of hydrocortisone was on average $20 \mathrm{mg}$ /day divided into two to three dosages. After withdrawal of hydrocortisone replacement for $24 \mathrm{~h}$, a fasting morning blood sample was taken for the measurement of serum cortisol concentrations. Patients with serum cortisol concentration $<120 \mathrm{nmol} / \mathrm{l}$ were considered to have ongoing glucocorticoid dependency, and hydrocortisone treatment was restarted. Patients with serum cortisol levels of 120-500 nmol/l were tested by ACTH stimulation tests $(250 \mu \mathrm{g})$. A normal response to ACTH stimulation was defined as a stimulated cortisol $>550 \mathrm{nmol} / \mathrm{l}$. When the cortisol response to ACTH was normal, patients were tested by insulin tolerance test (ITT) or CRH 
stimulation test. When cortisol responses to these tests were $<550 \mathrm{nmol} / \mathrm{l}$, hydrocortisone treatment was restarted. Evaluation of growth hormone $(\mathrm{GH})$ deficiency was done by insulin-tolerance test or arginineGHRH test only in patients under the age of 70 years and only after at least 2 years of remission. Patients with an inadequate stimulation of $\mathrm{GH}$ by one of these tests were treated with recombinant human $\mathrm{GH}$, aiming at insulin-like growth factor 1 levels between 0 and +2 S.D. values. In addition, the twice yearly evaluation consisted of measurement of free thyroxine and testosterone levels (in male patients). If results were below the lower limit of the respective reference ranges, L-thyroxine and/or testosterone substitution was started. In the case of amenorrhea and low estradiol levels in premenopausal women, estrogen replacement was provided. Persistent cure of CD was documented by normal values of a dexamethasone (1 mg) suppression test, urinary cortisol excretion rates, and midnight salivary cortisol levels before participation in the current study.

The estimated duration of disease was determined through patients' history by looking for the earliest physical/somatic signs. Duration of remission was calculated from the date of curative transsphenoidal surgery, or in case of persistent disease, from the date of normalization of biochemical tests after postoperative radiotherapy. Patient and treatment characteristics were collected from the patient records.

Written informed consent was obtained from all participants before the clinical assessment and the MRIscan session. Our institutional review board approved the study protocol. This study was in accordance with the principles of the declaration of Helsinki.

\section{Study design}

We scheduled a single study visit of $\sim 2 \mathrm{~h}$ for MRI scanning (60 min) and an interview for the evaluation of the clinical data and the assessment of psychological and cognitive functioning. Scan sessions took place between 0900 and $1200 \mathrm{~h}$. After the examination, participants were asked to complete several self-rating questionnaires at home for the assessment of psychopathology and cognitive functioning and to return them within a week.

\section{Assessment of psychopathology and cognitive functioning}

Presence and severity of depressive symptoms were evaluated using the Montgomery-Åsberg Depression Rating Scale (MADRS) $(24,25)$, which was the only scale that was assessed by the interviewer, and the Inventory of Depression Symptomatology (IDS) (26) (Supplementary information, see section on supplementary data given at the end of this article). Anxiety was evaluated using the Beck Anxiety Inventory (BAI)
(27) and the Fear Questionnaire (FQ) (28). Apathy and irritability were assessed using the Apathy Scale (AS) and the Irritability Scale (IS) respectively $(29,30)$. The Cognitive Failures Questionnaire (CFQ) was used to assess failures in perception, memory, and motor function (31).

\section{Cushing's syndrome severity index}

The Cushing's syndrome severity index (CSI) (32) was used to assess current severity of symptoms and to retrospectively estimate (clinical) severity at the time of active disease. The CSI contains eight clinical features and can be scored on a 3-point scale, ranging from 0 to 2. A higher total score on the CSI indicates greater severity, with a range of $0-16$. The information necessary for completing this index was derived from clinical history and medical files. Two raters, who reached consensus on each feature in case of discrepancy, scored the CSI. For the active phase, the CSI was scored retrospectively. The current score was evaluated based on the last yearly evaluation. The total score of the active phase and the total score of the remission phase were used in the analyses.

\section{MRI data acquisition}

Images were acquired on a Philips 3T MRI system (Philips Healthcare, Best, The Netherlands; software version 3.2.1). A SENSE-32 channel head coil was used for radio frequency transmission and reception. For each subject, anatomical images were obtained using a sagittal three-dimensional gradient-echo $\mathrm{T}_{1}$-weighted sequence (repetition time $=9.8 \mathrm{~ms}$, echo time $=4.6 \mathrm{~ms}$, matrix size $256 \times 256$, voxel size $1.17 \times 1.17 \times$ $1.2 \mathrm{~mm}, 140$ slices, scan duration $4: 56 \mathrm{~min}$ ) as part of a larger imaging protocol. A neuroradiologist, blinded for the clinical details of the subjects, examined all anatomical images. Apart from incidental age-related white matter hyperintensities and effects of the posttranssphenoidal surgery in the perisellar area, no other macroscopic abnormalities were observed in the patients and controls.

\section{Statistical analyses and data preprocessing}

The first analysis comprised the voxel-based comparison of grey matter volumes in the ROI (i.e. hippocampus, amygdala, and ACC) and across the whole brain between patients with predominantly long-term remission of $\mathrm{CD}$ and their matched healthy controls. Structural data were analyzed with FSL-VBM, a voxelbased morphometry style analysis (FMRIB's Software Library) (33). First, structural images were brainextracted and grey matter-segmented (34). The resulting grey matter partial volume images were then aligned to MNI-152 ( $\mathrm{T}_{1}$ standard brain average over 
152 subjects; Montreal Neurological Institute, Montreal, QC, Canada) standard space, using affine registration (35), followed by nonlinear registration. The resulting images of all participants were averaged to create a study-specific template, to which the native grey matter images were then nonlinearly reregistered.

The Jacobian of the warp field obtained in this registration reflects the voxel-wise relative volume change between the original and the study-specific template (i.e. a Jacobian of 5 indicates that a volume in the original image has been shrunk by a factor of 5). In order to correct for local expansion or contraction, the registered partial volume images were then modulated by dividing by the Jacobian of the warp field. The modulated segmented images were then smoothed with an isotropic Gaussian kernel with a sigma of $3 \mathrm{~mm}$. The Gaussian outputs a weighted average of each voxel's neighborhood, with the average weighted more toward the value of the centrally located voxels. The application of this type of smoothing reduces the noise in the data substantially.

The Harvard-Oxford Cortical and Subcortical Structural Atlases implemented in FSL were used to create masks for our ROI: the bilateral hippocampus, the bilateral amygdala, and the ACC. Probability range was set to $50-100 \%$ for all three structures. The studyspecific template was then applied to this mask to create a study-specific template of the grey matter values in the ROI only. Finally, groups were compared using a general linear model (GLM) including age, gender, and level of education as confound regressors. A voxel-wise GLM was applied using permutation-based (5000 permutations) non-parametric testing, correcting for multiple comparisons across space. First, groups were compared in our ROI, using the created mask. Second, an exploratory whole brain VBM analysis was done using the study-specific grey matter image as a mask to investigate whether any unpredicted differences existed between CD patients and controls. To explore possible differences between patients with hydrocortisone substitution $(n=13)$ and patients without substitution $(n=12)$, these two steps were repeated contrasting these two groups. Threshold-free Cluster Enhancement was used for finding clusters in the data (36), with thresholds for both the ROI comparison as well as the whole brain analysis set on $P<0.05$ corrected. In addition to the VBM analysis, we used FMRIB's integrated registration and segmentation tool (FIRST) to perform an automated segmentation of the amygdala and the hippocampus, allowing both shape and volume analyses.

The second analysis compared patients with predominantly long-term remission of $\mathrm{CD}$ and their matched healthy controls on measures of psychological and cognitive functioning. Data from questionnaires were analyzed using SPSS for Windows version 20.0 (SPSS, Inc.). All data are presented as numbers and percentages, means and S.D.s, or median and interquartile range (IQR). The assumption of normal distribution was tested using the Kolmogorov-Smirnov test and the assumption of equal variances with a Levene's test. With respect to psychological and cognitive functioning, normally distributed continuous variables between patients and matched controls were compared using $t$-tests, and non-normally distributed continuous variables (MADRS, IDS, BAI, FQ, AS, and CFQ) using Mann-Whitney $U$ tests. Considering the overlap in phenomenology assessed by the questionnaires, a strict correction for multiple testing might be too conservative, therefore all tests were two-sided with $P<0.05$ uncorrected.

A third analysis was conducted in the patient group. In this analysis, we examined voxel-wise correlations of behavioural and clinical characteristics with grey matter volume in the areas resulting from the ROI analysis and the whole brain analysis. The possible influence of radiotherapy could not be properly examined, considering the small number of patients that had received radiotherapy. The level of significance was set at $P<0.05$.

\section{Results \\ Patient characteristics}

As expected, patients and matched healthy controls did not differ in age, gender, or education. The mean estimated duration of active disease was $7.9 \pm 7.9$ years (range 0.8-37.0). The mean duration of remission was $11.2 \pm 8.2$ years (range 0.8-29.4). Hydrocortisone replacement therapy was given to 13 patients $(52 \%)$. The mean CSI score during active disease was $8.1 \pm 2.0$ and $2.5 \pm 1.5$ at the time of evaluation (i.e. long-term remission; Table 1).

\section{MRI analyses}

ROI analyses The VBM analysis, in patients in with predominantly long-term remission of $\mathrm{CD}$, showed smaller grey matter volumes in a large part of the bilateral ACC in comparison with controls. Closer examination of the data revealed that the patients had an average of $14 \%$ smaller grey matter volumes in the ACC compared with matched healthy controls. There were no grey matter volume differences in the bilateral hippocampus and amygdala (Fig. 1A). We observed no greater grey matter volumes in any of the ROIs in CD patients compared with controls. Furthermore, within the patient group no differences were found in grey matter volumes between patients with hydrocortisone substitution and patients without substitution. The FIRST analysis showed similar results, with no differences in both shape and volume of the bilateral amygdala and bilateral hippocampus, between patients and controls. 
Table 1 Clinical characteristics of patients with predominantly long-term remission of Cushing's disease $(n=25)$. Data are presented as mean \pm S.D. or number (\%) or by median IQR.

\begin{tabular}{lccc}
\hline & CD patients $(n=25)$ & Matched controls $(n=25)$ & $\boldsymbol{P}$ value \\
\hline Gender (male/female) & $4 / 21$ & $4 / 21$ & $1.000^{\mathrm{a}}$ \\
Age (years) & $45 \pm 8$ & $47 \pm 7$ & $0.471^{\mathrm{b}}$ \\
Education & $6(24 \%)$ & $6(24 \%)$ & $0.946^{\mathrm{a}}$ \\
Low & $12(48 \%)$ & $11(44 \%)$ & \\
Medium & $7(28 \%)$ & \\
High & $25(100 \%)$ & \\
Surgery & $2(8 \%)$ & \\
Transsphenoidal adenomectomy & $6(24 \%)$ & \\
Bilateral adrenalectomy & $7.9 \pm 7.9$ & \\
Radiotherapy & $11.2 \pm 8.2$ & \\
Disease duration (years) & $14(56 \%)$ & \\
Duration of remission (years) & $10(40 \%)$ & \\
Hypopituitarism & $9(36 \%)$ & \\
Any axis & $10(40 \%)$ & \\
GH & $3(12 \%)$ & \\
LH/FSH & $13(52 \%)$ & \\
TSH & $20.0(0.0-20.0)$ & \\
ADH & & \\
Hydrocortisone substitution & $8.1 \pm 2.0$ & \\
Hydrocortisone dose (mg/day) & $2.5 \pm 1.5$ & \\
Clinical severity index & & \\
Active phase (total) & & \\
Remission phase (total) & \\
\hline
\end{tabular}

$P$ values were tested with ${ }^{\mathrm{a}} \chi^{2}$ test and ${ }^{\mathrm{b}}$ independent-sample $t$-test.

Whole brain analysis Patients with predominantly long-term remission of CD showed smaller grey matter volumes in the left perigenual region (Brodman's area between BA 32 and BA 12) of the ACC, compared with controls (Fig. 1B). Greater grey matter volumes were found in the posterior lobe of the left cerebellum in CD patients compared with controls (Fig. 1C). On average patients showed 34\% larger grey matter volumes in the left posterior lobe of the cerebellum compared with controls $(P<0.05)$. When the threshold was lowered to $P<0.10$, an additional similar effect was observed in grey matter volumes of the right posterior lobe of the cerebellum. Within the patient group, no differences were found in grey matter volumes between patients with hydrocortisone substitution and patients without substitution.

\section{Psychopathology and cognitive functioning among patients and controls}

Table 2 shows that patients with predominantly longterm remission of $\mathrm{CD}$ had more depressive symptoms $(P<0.005)$ compared with controls, as assessed with the MADRS and the IDS. The mean total score on the MADRS was 6.3, indicating mild depressive symptoms. Furthermore, CD patients experienced more anxiety $(P=0.003)$, more social phobia $(P=0.034)$, and a greater degree of apathy $(P=0.002)$, with $44 \%$ of patients having a score of 14 or higher, which is indicative of clinically relevant apathy. On the IS, 36\% of the patients had a score of 14 or higher, which is indicative of clinically relevant irritability. In addition,
CD patients reported more cognitive failure $(P=0.023)$ compared with controls. No other significant betweengroup differences were found. Within the patient group, no significant differences were found in psychopathology and cognitive functioning between patients with hydrocortisone substitution and patients without substitution.

Furthermore, in the patient group no significant associations between grey matter volumes in the ACC and cerebellum, and scores on the distinguishing psychometric instruments (MADRS, IDS, BAI, AS, and CFQ), were found using a voxel-wise correlation approach. Also, no significant associations between grey matter volumes of the areas of effect and clinical characteristics (i.e. estimated disease duration, duration of remission, clinical severity indexes, active and remission subscale, data not shown) were found.

\section{Discussion}

This study demonstrates that structural abnormalities in the brain are present in patients cured from CD, despite long-term remission. The data indicate that in comparison with matched healthy controls, volumes of areas in the ACC were smaller, whereas grey matter volumes of the left posterior lobe of the cerebellum were larger in patients. There were no significant differences in grey matter volumes in the hippocampus or amygdala between the two groups. These findings may support the hypothesis that the increased prevalence of depressive symptoms, anxiety, apathy, and cognitive impairments observed in patients with 

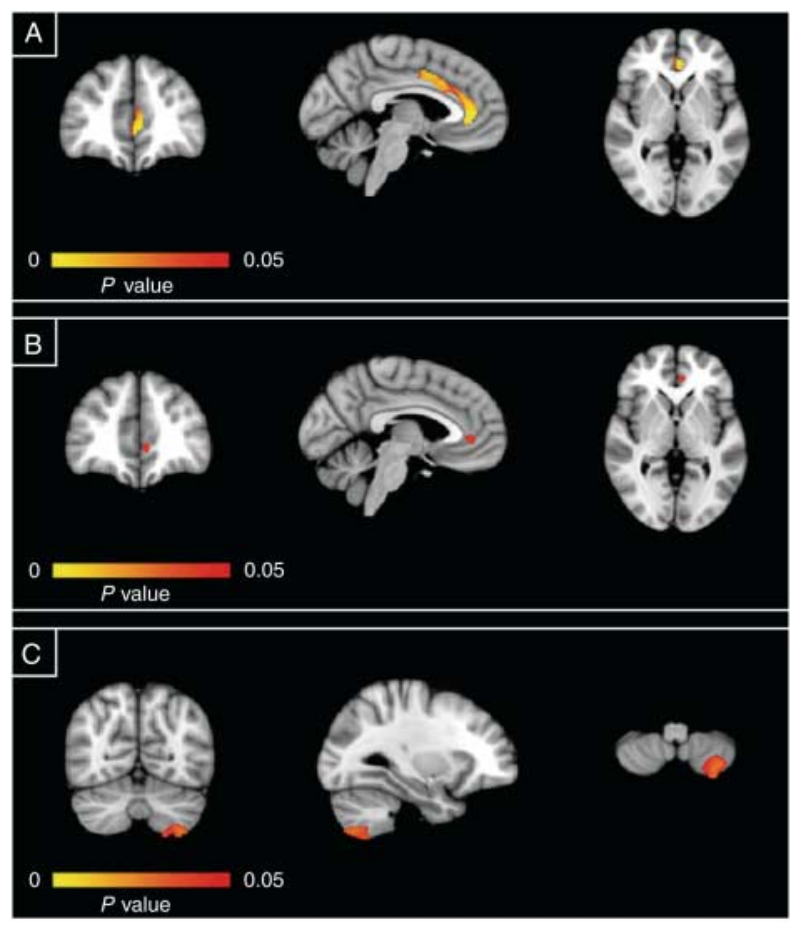

Figure 1 VBM analysis results. (A) Results of regions of interest analysis, with lesser grey matter volumes in patients than that in controls ( $P<0.05 ; 617$ voxels, $2 \mathrm{~mm}$ isotropic). (B) Results of whole brain analysis with lesser grey matter volumes in patients than that in controls ( $P<0.05$; 37 voxels, $2 \mathrm{~mm}$ isotropic). (C) Results of whole brain analysis with lesser grey matter volumes in patients than that in controls $(P<0.05 ; 323$ voxels, $2 \mathrm{~mm}$ isotropic). Effects are presented on the MNI-152 $1 \mathrm{~mm}$ standard brain at a threshold of $P<0.05$. Coordinates are $x=-4, y=42$, and $z=0$ for (A and $\mathrm{B}$ ) and $x=-29, y=-66$, and $z=-56$ for (C). The left hemisphere corresponds with the right side of the image.

long-term cured $\mathrm{CD}(22,23)$ is associated with structural brain changes. However, in these patients no significant correlations were found between psychological dysfunction and clinical characteristics on the one hand and the grey matter volumes of the ACC and left posterior lobe of the cerebellum on the other hand.

We confirmed our hypothesis that the ACC would be affected in cured CD. The amygdala and hippocampus are connected to the anterior regions of the ACC and constitute a neural circuitry for stress reactivity and modulation (37). Dysfunction of this circuitry is implicated in mood and anxiety disorders (38). In addition, patients with stress-related psychopathology show a reduced volume of the ACC $(39,40)$. In accordance, reduction of ACC volume is also found in animals exposed to hypercortisolism (8) and in elderly humans with dysregulation of the HPA-axis (10). Importantly, the ACC is involved in cognitive-affective processes, such as assessing the projection of emotional and motivational stimuli and the regulation of emotional responses (41), and mediates ongoing behavioral adaptation (42). Therefore, the identified abnormalities of the ACC may be involved in disturbances of cognitive and emotional functioning identified in CD (43) and in patients after long-term remission of $\mathrm{CD}(4,22,23)$. However, in the current study we were not able to demonstrate a correlation between the observed brain changes and quantitative estimates of psychopathology. This may be due to power problems or limitations of the clinical rating scales for psychopathology. An alternative hypothesis could be that the identified structural abnormalities may also underlie or reflect abnormalities in functional or structural connectivity.

In the exploratory whole brain analysis, we found an enlarged volume of the left cerebellum in patients with predominantly long-term remission of $\mathrm{CD}$. When we lowered the threshold, grey matter volumes of the right cerebellum was also found to be enlarged in patients with predominantly long-term remission of $\mathrm{CD}$, indicating that this effect might be bilateral. Interestingly, the cerebellum is susceptible to increased cortisol levels (44) and it is involved in motor functioning, as well as cognitive and emotional functioning (45). Intriguingly, a study by Spinelli et al. (46) reported that individuals exposed to an extremely stressful environment developed a larger cerebellum. Another research group investigated the effect of chronic stress on cortical and striatal circuits (required for goal-directed behavior and habits) in rats. They found global hypertrophy of the dorsolateral striatum and atrophy of the dorsomedial striatum and suggested that the reorganization of the corticostriatal circuits after chronic stress is bidirectional, based on hypertrophy and atrophy of neuronal dendritic trees (47). This mechanism of bidirectional reorganization could also provide an

Table 2 Psychopathology and cognitive failure questionnaires: patients with predominantly long-term remission of Cushing's disease vs matched controls. Data are presented as mean \pm s.D. or number (\%).

\begin{tabular}{lccc}
\hline & $\begin{array}{c}\text { Cushing's } \\
\text { disease } \\
(n=25)\end{array}$ & $\begin{array}{c}\text { Matched } \\
\text { controls } \\
(n=25)\end{array}$ & $\boldsymbol{P}$ value \\
\hline MADRS & $6.3 \pm 5.5$ & $1.4 \pm 1.8$ & $\mathbf{0 . 0 0 0}^{\mathrm{b}}$ \\
Inventory of Depression & $46.8 \pm 13.0$ & $36.3 \pm 5.8$ & $\mathbf{0 . 0 0 5}^{\mathrm{b}}$ \\
$\quad$ Symptomatology & $28.4 \pm 5.7$ & $24.0 \pm 3.1$ & $\mathbf{0 . 0 0 3}^{\mathrm{b}}$ \\
Beck Anxiety Inventory & $24.5 \pm 17.4$ & $14.2 \pm 10.0$ & $0.051^{\mathrm{b}}$ \\
Fear Questionnaire & $6.1 \pm 7.9$ & $3.4 \pm 4.7$ & $0.477^{\mathrm{b}}$ \\
Agoraphobia subscale & $6.2 \pm 8.3$ & $3.2 \pm 4.1$ & $0.118^{\mathrm{a}}$ \\
Blood injury phobia & & & \\
$\quad$ subscale & $12.2 \pm 8.0$ & $7.6 \pm 4.9$ & $\mathbf{0 . 0 3 4}^{\mathrm{b}}$ \\
Social phobia subscale & $12.1 \pm 8.7$ & $8.0 \pm 6.1$ & $0.066^{\mathrm{a}}$ \\
Irritability Scale & $9(36 \%)$ & $6(24 \%)$ & \\
$\quad$ Total score $>14$ & $13.6 \pm 6.6$ & $7.8 \pm 3.8$ & $\mathbf{0 . 0 0 2}^{\mathrm{b}}$ \\
Apathy Scale & $11(44 \%)$ & $2(8 \%)$ & \\
$\quad$ Total score $>14$ & $38.0 \pm 16.5$ & $27.6 \pm 9.7$ & $\mathbf{0 . 0 2 3}^{\mathrm{b}}$ \\
Cognitive Failures & & & \\
$\quad$ Questionnaire & & & \\
\hline
\end{tabular}

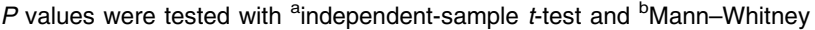
$U$ test. Level of significance was set at $P<0.05$ and significant values are in bold. 
explanation for the larger volume of the cerebellum in our patients treated for CD.

Contrary to our hypotheses, we did not find alterations in the hippocampus and amygdala. However, it might be that these brain structures were affected during active disease $(11,12,13)$, but that grey matter volumes increased after biochemical cure. This would be in accordance with the previously found increase in hippocampal volume in $\mathrm{CD}$ patients after correction of hypercortisolism $(11,14,15,16)$ and the well-documented plasticity of hippocampal neurons in animal models (48). Nevertheless, children experienced cognitive decline despite reversal of brain atrophy 1 year after surgical remission (49) and adult patients with long-term remission of CD still demonstrated impaired memory function (23). Recently, a potential mechanism was provided for this persisted memory impairment, by demonstrating that in comparison with healthy matched controls, patients in remission of CD show biochemical abnormalities in the hippocampus, without reduction in hippocampal volume (50). Studies on animals have documented that other brain areas also show structural changes in response to increased cortisol levels (47). However, the plasticity (in this case the extent of reversibility) of these nonhippocampal structures in CD is still unknown. As there are no studies that have focused on other brain structures in patients with active CD, like the ACC or amygdala, it is not clear when these structural changes occur or how they develop over time.

For direct effects of glucocorticoids on a brain area, either the MR or GR has to be present in this area. Using data on human brain tissue arrays available from The Allen Institute for Brain Science, a high expression was demonstrated of both MR and/or GR, not only in the hippocampus, ACC, and amygdala but also in the cerebellum (51). Taking into account the effects found in our study, which were limited to the ACC and cerebellum, one can conclude that expression of MR and/or GR in a brain area is necessary, but not predictive of structural changes following chronic overexposure to glucocorticoids. A possible alternative explanation is that structural changes may also occur via transsynaptic mechanisms. Such mechanisms have been suggested for (transient) morphological changes in the hippocampal CA3 area, which itself expresses very low numbers of GRs, but receives input from the cortisol-sensitive dentate gyrus (52).

To our knowledge, our study is the first to show that structural abnormalities in the brain are present in patients cured from $\mathrm{CD}$, despite long-term remission. Strengths of our study are the homogeneity of our patient cohort with regard to treatment (i.e. all patients had been treated with transsphenoidal surgery) and the careful selection of controls. Nevertheless, heterogeneity still existed in the patient group with regard to disease duration and duration of remission, which may have decreased the power of this study. Although a sample size of 25 in both groups is appropriate for the evaluation of structural changes with MRI (53), our study might have been underpowered to detect possible correlations between clinical data, psychological and cognitive measures, and grey matter volumes within the patient group, and to detect grey matter differences between patients with or without hydrocortisone substitution. In addition, cognitive functions were assessed using a questionnaire (i.e. CFQ), and although this questionnaire has been validated repeatedly, it is no substitute for extensive neuropsychological testing, which gives a more accurate representation of cognitive functioning. Furthermore, because of our cross-sectional design it cannot be excluded that structural abnormalities were already present in patients before onset of CD. The use of a longitudinal design in future research could provide more insight into the course of the found abnormalities.

In general, alterations in grey matter volume in adults with pathology have been found to be associated with dysfunctions of specific areas or related circuitry. However, the absence of volumetric differences does not exclude functional alterations in brain areas and circuits. It should also be acknowledged that a volumetric VBM approach does not reveal the underlying changes or pathology in grey matter microstructure, i.e. at the level of neurons or glia cells. Subsequently, at present there are no data available on abnormalities at the level of neurons or glia cells after chronic overexposure to glucocorticoids that may shed more light on the nature of the observed structural abnormalities. Therefore, conclusions about functional alterations in the specific brain areas cannot be drawn based solely on our findings. Exploring functional brain characteristics in our sample would be an important next step to further elucidate the neurobiological basis of psychological dysfunction in patients with remitted CD.

The data presented in this study provide a further perspective toward detailed phenotyping of patients after treatment of $\mathrm{CD}$, who have always been considered cured after long-term remission of hypercortisolism. In agreement with others, CD and possibly Cushing's syndrome as well, could be a unique model to study the apparently prolonged, or even irreversible, effects of increased cortisol exposure on the brain. It is tempting to speculate that these findings, to a certain extent, could also apply to patients with chronic or recurrent forms of highly prevalent stress-related disorders and, in addition, to patients chronically treated with exogenous corticosteroids that are commonly prescribed to suppress the immune system (54).

In summary, this study demonstrates that patients with long-term cure after treatment for $\mathrm{CD}$ have profound structural alterations in the brain, with smaller volumes of an area in the ACC and greater volumes of the left posterior lobe of the cerebellum, and report more depressive symptoms, anxiety, social 
phobia, apathy, and cognitive failure, compared with healthy controls. The findings suggest possible structural substrates for long-term psychological effects of hypercortisolemia in CD. Clearly, more research is needed to increase our insight into the underlying mechanisms and the trajectory of changes, which may also lead to the identification of 'critical time windows' or potential targets for prevention.

\section{Supplementary data}

This is linked to the online version of the paper at http://dx.doi.org/ 10.1530/EJE-13-0471.

\section{Declaration of interest}

The authors declare that there is no conflict of interest that could be perceived as prejudicing the impartiality of the research reported.

\section{Funding}

S J A van der Werff was supported through The Netherlands Organization for Scientific Research - National Initiative Brain and Cognition project (NWO-NIHC, project no. 056-25-010). N R Biermasz was supported through The Netherlands Organization for Scientific Research (NWO-VENI, project no. 016136125).

\section{References}

1 Newell-Price J, Bertagna X, Grossman AB \& Nieman LK. Cushing's syndrome. Lancet 2006367 1605-1617. (doi:10.1016/S01406736(06)68699-6)

2 Dorn LD, Burgess ES, Friedman TC, Dubbert B, Gold PW \& Chrousos GP. The longitudinal course of psychopathology in Cushing's syndrome after correction of hypercortisolism. Journal of Clinical Endocrinology and Metabolism 199782 912-919. (doi:10.1210/jc.82.3.912)

3 Starkman MN, Schteingart DE \& Schork MA. Cushing's syndrome after treatment: changes in cortisol and ACTH levels, and amelioration of the depressive syndrome. Psychiatry Research 198619 177-188. (doi:10.1016/0165-1781(86)90096-X)

4 Pereira AM, Tiemensma J \& Romijn JA. Neuropsychiatric disorders in Cushing's syndrome. Neuroendocrinology 201092 (Suppl 1) 65-70. (doi:10.1159/000314317)

5 Feelders RA, Pulgar SJ, Kempel A \& Pereira AM. Management of endocrine disease: the burden of Cushing's disease: clinical and health-related quality of life aspects. European Journal of Endocrinology 2012167 311-326. (doi:10.1530/EJE-11-1095)

6 De Kloet ER, Joels M \& Holsboer F. Stress and the brain: from adaptation to disease. Nature Reviews. Neuroscience 20056 463-475. (doi:10.1038/nrn1683)

7 McEwen BS, Eiland L, Hunter RG \& Miller MM. Stress and anxiety: structural plasticity and epigenetic regulation as a consequence of stress. Neuropharmacology 201262 3-12. (doi:10.1016/j.neuropharm.2011.07.014)

8 Cerqueira JJ, Catania C, Sotiropoulos I, Schubert M, Kalisch R, Almeida OF, Auer DP \& Sousa N. Corticosteroid status influences the volume of the rat cingulate cortex - a magnetic resonance imaging study. Journal of Psychiatric Research 200539 451-460. (doi:10.1016/j.jpsychires.2005.01.003)

9 Lupien SJ, de Leon M, de Santi S, Convit A, Tarshish C, Nair NP, Thakur M, McEwen BS, Hauger RL \& Meaney MJ. Cortisol levels during human aging predict hippocampal atrophy and memory deficits. Nature Neuroscience 19981 69-73. (doi:10.1038/271)
10 MacLullich AM, Ferguson KJ, Wardlaw JM, Starr JM, Deary IJ \& Seckl JR. Smaller left anterior cingulate cortex volumes are associated with impaired hypothalamic-pituitary-adrenal axis regulation in healthy elderly men. Journal of Clinical Endocrinology and Metabolism 200691 1591-1594. (doi:10.1210/jc.20052610)

11 Bourdeau I, Bard C, Noel B, Leclerc I, Cordeau MP, Belair M, Lesage J, Lafontaine L \& Lacroix A. Loss of brain volume in endogenous Cushing's syndrome and its reversibility after correction of hypercortisolism. Journal of Clinical Endocrinology and Metabolism 200287 1949-1954. (doi:10.1210/jc.87.5. 1949)

12 Starkman MN, Gebarski SS, Berent S \& Schteingart DE. Hippocampal formation volume, memory dysfunction, and cortisol levels in patients with Cushing's syndrome. Biological Psychiatry 199232 756-765. (doi:10.1016/0006-3223(92)90079-F)

13 Simmons NE, Do HM, Lipper MH \& Laws ER Jr. Cerebral atrophy in Cushing's disease. Surgical Neurology $2000 \quad 53 \quad 72-76$. (doi:10.1016/S0090-3019(99)00197-4)

14 Toffanin T, Nifosi F, Follador H, Passamani A, Zonta F, Ferri G, Scanarini M, Amista P, Pigato G, Scaroni C et al. Volumetric MRI analysis of hippocampal subregions in Cushing's disease: a model for glucocorticoid neural modulation. European Psychiatry 2011 26 64-67. (doi:10.1016/j.eurpsy.2010.09.003)

15 Starkman MN, Giordani B, Gebarski SS, Berent S, Schork MA \& Schteingart DE. Decrease in cortisol reverses human hippocampal atrophy following treatment of Cushing's disease. Biological Psychiatry 199946 1595-1602. (doi:10.1016/S0006-3223 (99)00203-6)

16 Hook JN, Giordani B, Schteingart DE, Guire K, Giles J, Ryan K, Gebarski SS, Langenecker SA \& Starkman MN. Patterns of cognitive change over time and relationship to age following successful treatment of Cushing's disease. Journal of the International Neuropsychological Society 200713 21-29. (doi:10.1017/ S1355617707070051)

17 Starkman MN, Giordani B, Gebarski SS \& Schteingart DE. Improvement in learning associated with increase in hippocampal formation volume. Biological Psychiatry 200353 233-238. (doi:10.1016/S0006-3223(02)01750-X)

18 Starkman MN, Giordani B, Gebarski SS \& Schteingart DE. Improvement in mood and ideation associated with increase in right caudate volume. Journal of Affective Disorders 2007101 139-147. (doi:10.1016/j.jad.2006.11.007)

19 Resmini E, Santos A, Gomez-Anson B, Vives Y, Pires P, Crespo I, Portella MJ, de Juan-Delago M, Barahona MJ \& Webb SM. Verbal and visual memory performance and hippocampal volumes, measured by 3-Tesla magnetic resonance imaging, in patients with Cushing's syndrome. Journal of Clinical Endocrinology and Metabolism 201297 663-671. (doi:10.1210/jc.2011-2231)

20 van Aken MO, Pereira AM, Biermasz NR, van Thiel SW, Hoftijzer HC, Smit JW, Roelfsema F, Lamberts SW \& Romijn JA. Quality of life in patients after long-term biochemical cure of Cushing's disease. Journal of Clinical Endocrinology and Metabolism 200590 3279-3286. (doi:10.1210/jc.2004-1375)

21 Tiemensma J, Kaptein AA, Pereira AM, Smit JW, Romijn JA \& Biermasz NR. Negative illness perceptions are associated with impaired quality of life in patients after long-term remission of Cushing's syndrome. European Journal of Endocrinology 2011165 527-535. (doi:10.1530/EJE-11-0307)

22 Tiemensma J, Biermasz NR, Middelkoop HA, van der Mast RC, Romijn JA \& Pereira AM. Increased prevalence of psychopathology and maladaptive personality traits after long-term cure of Cushing's disease. Journal of Clinical Endocrinology and Metabolism 201095 E129-E141. (doi:10.1210/jc.2010-0512)

23 Tiemensma J, Kokshoorn NE, Biermasz NR, Keijser BJ, Wassenaar MJ, Middelkoop HA, Pereira AM \& Romijn JA. Subtle cognitive impairments in patients with long-term cure of Cushing's disease. Journal of Clinical Endocrinology and Metabolism 201095 2699-2714. (doi:10.1210/jc.2009-2032) 
24 Montgomery SA \& Asberg M. A new depression scale designed to be sensitive to change. British Journal of Psychiatry 1979134 382-389. (doi:10.1192/bjp.134.4.382)

25 Snaith RP, Harrop FM, Newby DA \& Teale C. Grade scores of the Montgomery-Asberg Depression and the Clinical Anxiety Scales. British Journal of Psychiatry 1986148 599-601. (doi:10.1192/ bjp.148.5.599)

26 Rush AJ, Gullion CM, Basco MR, Jarrett RB \& Trivedi MH. The Inventory of Depressive Symptomatology (IDS): psychometric properties. Psychological Medicine $1996 \quad 26 \quad 477-486$. (doi:10.1017/SO033291700035558)

27 Beck AT, Epstein N, Brown G \& Steer RA. An inventory for measuring clinical anxiety: psychometric properties. Journal of Consulting and Clinical Psychology $1988 \quad 56 \quad 893-897$. (doi:10.1037/0022-006X.56.6.893)

28 Marks IM \& Mathews AM. Brief standard self-rating for phobic patients. Behaviour Research and Therapy 197917 263-267. (doi:10.1016/0005-7967(79)90041-X)

29 Starkstein SE, Petracca G, Chemerinski E \& Kremer J. Syndromic validity of apathy in Alzheimer's disease. American Journal of Psychiatry 2001158 872-877. (doi:10.1176/appi.ajp.158.6.872)

30 Chatterjee A, Anderson KE, Moskowitz CB, Hauser WA \& Marder KS. A comparison of self-report and caregiver assessment of depression, apathy, and irritability in Huntington's disease. Journal of Neuropsychiatry and Clinical Neurosciences 200517 378-383. (doi:10.1176/appi.neuropsych.17.3.378)

31 Broadbent DE, Cooper PF, FitzGerald P \& Parkes KR. The Cognitive Failures Questionnaire (CFQ) and its correlates. British Journal of Clinical Psychology 198221 1-16. (doi:10.1111/j.2044-8260. 1982.tb01421.x)

32 Sonino N, Boscaro M, Fallo F \& Fava GA. A clinical index for rating severity in Cushing's syndrome. Psychotherapy and Psychosomatics 200069 216-220. (doi:10.1159/000012396)

33 Smith SM, Jenkinson M, Woolrich MW, Beckmann CF, Behrens TE, Johansen-Berg H, Bannister PR, De Luca M, Drobnjak I, Flitney DE et al. Advances in functional and structural MR image analysis and implementation as FSL. NeuroImage 200423 (Suppl 1) S208-S219. (doi:10.1016/j.neuroimage.2004.07.051)

34 Zhang Y, Brady M \& Smith S. Segmentation of brain MR images through a hidden Markov random field model and the expectation-maximization algorithm. IEEE Transactions on Medical Imaging 200120 45-57. (doi:10.1109/42.906424)

35 Jenkinson M, Bannister P, Brady M \& Smith S. Improved optimization for the robust and accurate linear registration and motion correction of brain images. NeuroImage $2002 \mathbf{1 7}$ 825-841. (doi:10.1006/nimg.2002.1132)

36 Smith SM \& Nichols TE. Threshold-free cluster enhancement: addressing problems of smoothing, threshold dependence and localisation in cluster inference. NeuroImage $2009 \mathbf{4 4} 83-98$. (doi:10.1016/j.neuroimage.2008.03.061)

37 Shin LM \& Liberzon I. The neurocircuitry of fear, stress, and anxiety disorders. Neuropsychopharmacology 201035 169-191. (doi:10.1038/npp.2009.83)

38 Bremner JD. Neuroimaging in posttraumatic stress disorder and other stress-related disorders. Neuroimaging Clinics of North America 200717 523-538, ix. (doi:10.1016/j.nic.2007.07.003)

39 Woodward SH, Kaloupek DG, Streeter CC, Martinez C, Schaer M \& Eliez S. Decreased anterior cingulate volume in combat-related PTSD. Biological Psychiatry 200659 582-587. (doi:10.1016/ j.biopsych.2005.07.033)

40 van Tol MJ, van der Wee NJ, van den Heuvel OA, Nielen MM, Demenescu LR, Aleman A, Renken R, van Buchem MA, Zitman FG \& Veltman DJ. Regional brain volume in depression and anxiety disorders. Archives of General Psychiatry 201067 1002-1011. (doi:10.1001/archgenpsychiatry.2010.121)
41 Bush G, Luu P \& Posner MI. Cognitive and emotional influences in anterior cingulate cortex. Trends in Cognitive Sciences $2000 \mathbf{4}$ 215-222. (doi:10.1016/S1364-6613(00)01483-2)

42 Sheth SA, Mian MK, Patel SR, Asaad WF, Williams ZM, Dougherty DD, Bush G \& Eskandar EN. Human dorsal anterior cingulate cortex neurons mediate ongoing behavioural adaptation. Nature $2012 \mathbf{4 8 8} 218-221$. (doi:10.1038/nature11239)

43 Starkman MN \& Schteingart DE. Neuropsychiatric manifestations of patients with Cushing's syndrome. Relationship to cortisol and adrenocorticotropic hormone levels. Archives of Internal Medicine 1981141 215-219. (doi:10.1001/archinte.1981.003400 20077021)

44 Teicher MH, Andersen SL, Polcari A, Anderson CM, Navalta CP \& Kim DM. The neurobiological consequences of early stress and childhood maltreatment. Neuroscience and Biobehavioral Reviews 200327 33-44. (doi:10.1016/S0149-7634(03)00007-1)

45 Baumann $\mathrm{O} \&$ Mattingley JB. Functional topography of primary emotion processing in the human cerebellum. NeuroImage 2012 61 805-811. (doi:10.1016/j.neuroimage.2012.03.044)

46 Spinelli S, Chefer S, Suomi SJ, Higley JD, Barr CS \& Stein E. Early-life stress induces long-term morphologic changes in primate brain. Archives of General Psychiatry 200966 658-665. (doi:10.1001/archgenpsychiatry.2009.52)

47 Dias-Ferreira E, Sousa JC, Melo I, Morgado P, Mesquita AR, Cerqueira JJ, Costa RM \& Sousa N. Chronic stress causes frontostriatal reorganization and affects decision-making. Science 2009325 621-625. (doi:10.1126/science.1171203)

48 Schubert MI, Kalisch R, Sotiropoulos I, Catania C, Sousa N, Almeida OF \& Auer DP. Effects of altered corticosteroid milieu on rat hippocampal neurochemistry and structure - an in vivo magnetic resonance spectroscopy and imaging study. Journal of Psychiatric Research 200842 902-912. (doi:10.1016/j.jpsychires.2007.10.003)

49 Merke DP, Giedd JN, Keil MF, Mehlinger SL, Wiggs EA, Holzer S, Rawson E, Vaituzis AC, Stratakis CA \& Chrousos GP. Children experience cognitive decline despite reversal of brain atrophy one year after resolution of Cushing syndrome. Journal of Clinical Endocrinology and Metabolism 200590 2531-2536. (doi:10.1210/jc.2004-2488)

50 Resmini E, Santos A, Gomez-Anson B, Lopez-Mourelo O, Pires P, Vives-Gilabert Y, Crespo I, Portella MJ, de Juan-Delago M \& Webb SM. Hippocampal dysfunction in cured Cushing's syndrome patients, detected by $1 \mathrm{H}-\mathrm{MR}$-spectroscopy. Clinical Endocrinology 2013. In press. (doi:10.1111/cen.12224)

51 Hawrylycz MJ, Lein ES, Guillozet-Bongaarts AL, Shen EH, Ng L, Miller JA, van de Lagemaat LN, Smith KA, Ebbert A, Riley ZL et al. An anatomically comprehensive atlas of the adult human brain transcriptome. Nature $2012 \mathbf{4 8 9}$ 391-399. (doi:10.1038/ nature11405)

52 Fuchs E, Flugge G \& Czeh B. Remodeling of neuronal networks by stress. Frontiers in Bioscience 200611 2746-2758. (doi:10.2741/ 2004)

53 Pell GS, Briellmann RS, Chan CH, Pardoe H, Abbott DF \& Jackson GD. Selection of the control group for VBM analysis: influence of covariates, matching and sample size. NeuroImage 200841 1324-1335. (doi:10.1016/j.neuroimage.2008.02.050)

54 Brown ES \& Suppes T. Mood symptoms during corticosteroid therapy: a review. Harvard Review of Psychiatry 1998 5 239-246. (doi:10.3109/10673229809000307)

Received 5 June 2013

Revised version received 6 September 2013

Accepted 12 September 2013 